\title{
Self-Sexual de jovens adultos brasileiros
}

\author{
Self-Sexual de jóvenes adultos brasileños \\ Sexual-Self of Young Brazilian Adults
}

\author{
Leo S. de Azevedo e Souza* \\ Mariana G. Boeckel \\ Universidade Federal de Ciências da Saúde de Porto Alegre
}

Doi: https://doi.org/10.12804/revistas.urosario.edu.co/apl/a.7523

\section{Resumo}

Self-sexual é um constructo auto percebido multifacetado, que inclui eficácia sexual, autoestima sexual-corporal, sensação de direito a receber prazer de si e do(a) parceiro(a), e autorreflexão-sexual. O presente estudo investigou o self-sexual de jovens adultos brasileiros, avaliando a correlação entre self-sexual e as escalas de satisfação com a vida e de autoestima geral, assim como o impacto da religiosidade no self-sexual. 1380 participantes, de 18 a 30 anos, completaram uma pesquisa online que incluiu o Inventário de Self-sexual (ISS). O delineamento foi quantitativo, descritivo e transversal. Todas subescalas do ISS obtiveram correlação positiva com as escalas de satisfação com a vida e de autoestima geral. Algumas subescalas apresentaram correlação negativa com religiosidade, embora a subescala de autoestima sexual tenha obtido correlação positiva. As mulheres obtiveram escores significativamente maiores nas subescalas de sensação de direito a receber prazer de si e do(a) outro(a). As mulheres bissexuais se destacaram com maiores resultados em comparação a homens e mulheres heterossexuais, apesar de apresentarem menores níveis de autoestima. Pesquisas futuras com variáveis e amostras mais diversas podem contribuir para a continuidade da compreensão desse constructo.

Palavras-chave: saúde sexual, sexualidade, autoestima.

\section{fbstract}

Sexual subjectivity (translated as sexual-self once studied in Brazil) is a self-perceptive multi-layered construct, which includes sexual efficacy, sexual body-esteem, entitlement to sexual pleasure from self and partner, and sexual self-reflection. This study investigated the sexual subjectivity of young Brazilian adults, evaluated the correlation between sexual subjectivity and quality of life, and also the impact of religiosity on sexual subjectivity. Participants were 1380 from ages 18 to 30 who completed a free online survey, including the Inventário de Self-Sexual (ISS). The design was quantitative, descriptive, and transversal. All of the Iss's subscales had a positive correlation with the satisfaction with life and self-esteem scales. Some sexual subjectivity subscales showed a negative correlation with religiosity, while sexual body-esteem presented a positive

* Dirigir correspondencia a Leo S. de Azevedo e Souza: Faculdade de Psicologia, Universidade Federal de Ciências da Saúde de Porto Alegre. Correio eletrônico: leoazevedoesouza@gmail.com 
one. The women in the sample scored significantly higher on the entitlement to sexual pleasure from self and partner subscales. Bisexual women stood out with higher scores than the heterosexual men and women, despite having lower self-esteem. Future research with more variables and a broader sample will help further the understanding of this barely explored construct. Keywords: Sexual health, sexuality, self-esteem.

\section{Resumen}

Self-sexual es un constructo auto percibido multifacético, que incluye eficacia sexual, autoestima sexual-corporal, sensación de derecho a recibir placer de sí y de su pareja, y autorreflexión sexual. El presente estudio investigó el Self-sexual de adultos jóvenes brasileros, evaluando la correlación entre este y las escalas de satisfacción con la vida y de autoestima general, así como el impacto de la religiosidad en el Self-sexual. 1380 participantes, de 18 a 30 años, completaron una encuesta en línea que incluyó el Inventario de Self-Sexual (ISS). El delineamiento fue cuantitativo, descriptivo y transversal. Todas las subescalas del ISS obtuvieron correlación positiva con las escalas de satisfacción con la vida y de autoestima general. Algunas subescalas presentaron correlación negativa con religiosidad, a pesar de que la subescala de autoestima sexual obtuvo una correlación positiva. Las mujeres de la muestra obtuvieron puntajes significativamente mayores en las subescalas de sensación de derecho a recibir placer de sí y de otro. Las mujeres bisexuales se destacaron con mayores resultados que hombres y mujeres heterosexuales, a pesar de presentar menores niveles de autoestima. Investigaciones futuras con variables y muestras más diversas colaboraron para la secuencia de comprensión de ese constructo.

Palabras clave: salud sexual, sexualidad, autoestima.

O self-sexual diz respeito a um conjunto de percepções cognitivas que uma pessoa possui a respeito de sua própria sexualidade. A maneira como ela se vê e se aceita enquanto um ser sexual
(Horne \& Zimmer-Gembeck, 2006). Considere um espectro no qual, em uma extremidade, temos uma pessoa que se vê apropriada e à vontade com sua sexualidade, sendo um agente ativo e autônomo nas suas escolhas sexuais. Na outra extremidade, uma pessoa que se sente estranha com sua sexualidade, sem saber o que quer e pressionada por expectativas e padrões externos. Quando examinamos o self-sexual de alguém, estamos avaliando um constructo subjetivo em que a pessoa terá um grau de apropriação e autoaceitação da sua identidade sexual. Na definição de Martin (1996), a apropriação do self-sexual é um componente necessário de atitude (sentir-se ativo) e, portanto, de autoestima. Ou seja, a sexualidade de alguém afeta sua capacidade de atuar no mundo, e de sentir que pode desejar coisas e fazê-las acontecerem. No entanto, deve-se questionar: o que pode favorecer com que uma pessoa tenha mais atitude sobre sua identidade sexual? Isto é, que variáveis possuem um impacto significativo no self-sexual?

Em relação aos estudos do self-sexual, poucos pesquisadores exploraram tal conceito, destacando-se os trabalhos de Horne e Zimmer-Gembeck (2006) e Zimmer-Gembeck e French (2016), que investigaram, na população jovem da Austrália, quais componentes poderiam ser mais influentes sobre o self-sexual. Cinco componentes, mensurados individualmente, foram estabelecidos como os pilares do self-sexual, sendo eles: autoestima corporal-sexual; sensação de direito a receber prazer sexual de si mesmo(a); sensação de direito a receber prazer sexual do(a) parceiro(a); autoeficácia para alcançar o prazer sexual e a autorreflexão sexual (Horne \& Zimmer-Gembeck, 2006).

\section{Autoestima corporal-sexual}

Este componente diz respeito à relação de compreensão e aceitação de uma pessoa com seu próprio corpo e sua imagem (Martin, 1996), sua identificação com seu gênero e o grau de valor 
dado a esse corpo - sobre o qual parâmetros sociais podem ser impactantes (Amos \& McCabe, 2016).

\section{Sensação de direito a receber prazer sexual de si mesmo, sensação de direito a receber prazer sexual do(a) parceiro(a) e autoeficácia sexual}

A maneira com que o prazer sexual é experienciado também é um componente do self-sexual. Desejo sexual possui, além dos aspectos físicos, componentes cognitivos e emocionais ligados ao reconhecimento de vontades e fantasias sexuais (Ellis, 2004; Giner-Sorolla, Bosson, Caswell, \& Hettinger, 2012; Martin, 1996). A capacidade de identificar tais desejos - e, principalmente, de aceitá-los moralmente - proporciona uma liberdade para manifestar essa excitação ao explorar o próprio corpo, permitindo que a pessoa receba prazer sexual de si mesma, sendo a masturbação a principal representação do prazer vindo de si (Baumel, 2014; Michaud, Suris \& Deppen, 2006). Em outras palavras, se houver uma aceitação - amparada por questões culturais e sociais (incluindo o impacto da religião) - de que a pessoa possui o direito de receber prazer sexual de si mesmo, maior será a sensação de que é ela quem está tomando suas próprias decisões e incorporando sua sexualidade de maneira ativa e sem culpa (Baumel, 2014; Horne \& Zimmer-Gembeck, 2006).

Neste mesmo processo se enquadra a sensação de direito a receber prazer sexual do(a) parceiro(a), porém desta vez outras variáveis ganham relevância, uma vez que há outra pessoa envolvida, como por exemplo, perceber-se menos experiente sexualmente do que o parceiro(a), sentir-se seguro(a) e ser merecedor deste prazer (Hewitt-Stubbs, Zimmer-Gembeck, Mastro \& Boislard, 2016). Por fim, a Autoeficácia Sexual diz respeito à capacidade de atingir o prazer sexual e de proporcionar os meios para tal, incluindo, dentre os múltiplos comportamentos sexuais, a capacidade de comunicar seus desejos (Horne
\& Zimmer-Gembeck, 2006; Zimmer-Gembeck \& French, 2016).

\section{Autorreflexão sexual}

O quinto pilar do self-sexual é a autorreflexão sexual. Assim como nos outros componentes do self-sexual, a aceitação terá um papel de destaque na autorreflexão sexual, porém, neste caso, trata-se de uma capacidade de lidar com experiências vivenciadas e refletir sobre elas de maneira a aprender com elas para situações futuras (Horne \& Zimmer-Gembeck, 2006). Ao avaliar experiências da sua vida sexual, uma pessoa pode julgá-las com culpa e arrependimento, demonstrando preocupação com sua reputação ou com as consequências sociais. No outro extremo do espectro veremos alguém assumindo responsabilidade pela decisão dos seus atos, aceitando o que se passou como aprendizado do que quer (ou não quer) voltar a experienciar. Desta forma, a autorreflexão sexual tem o potencial de capacitar as pessoas a exercerem uma compreensão da maneira e do porquê de agirem como agem - tornando-se, assim, agentes ativos nas suas futuras decisões sexuais.

\section{Gênero e diversidade sexual}

Ao pesquisarem diferenças de gênero em alguns dos componentes da subjetividade sexual, Zimmer-Gembeck e French (2016) encontraram que homens cisgêneros reportaram maior sensação de direito a receber prazer deles mesmos e maior autoeficácia para alcançar o prazer sexual, enquanto mulheres cisgêneras reportaram maior sensação de direito a receber prazer sexual do(a) parceiro(a) (as demais não apresentaram diferenças significativas). Tais resultados podem ser consequência de as mulheres serem culturalmente menos incentivadas a identificarem e comunicarem seus desejos sexuais (Impett \& Peplau, 2003; Tolman \& Tolman, 2009). A partir deste resultado, é possível especular que, embora 
o processo comunicativo de manifestar desejos e preferências seja complicado (em particular com novos parceiros), as mulheres se sentem no direito de receber esse prazer, tendo como desafio capacitar seus parceiros a lhes agradarem. No entanto, os resultados supracitados dizem respeito à população cisgênera (pessoas que se identificam com o sexo designado a elas), que é também heterossexual na sua vasta maioria. É possível que pessoas que pertençam a grupos minoritários no que tange à identidade de gênero e/ou orientação sexual apresentem escores diferentes na escala de self-sexual. Considerando que a autoestima e o bem-estar desta população tendem a ser relacionados à percepção da discriminação sexual (Greene \& Britton, 2013), é possível que haja alguma discrepância entre as pessoas de diferentes identidades de gênero e orientações sexuais no que diz respeito aos níveis de subjetividade sexual.

\section{Religiosidade}

Outra variável que pode impactar a subjetividade sexual é a religiosidade. Indivíduos religiosos, seja exercendo sua religiosidade de maneira intrínseca ou extrínseca, tendem a ter menos atividade sexual (Moreau, Trussell \& Bajos, 2013; Zaleski \& Schiaffino, 2000). Em um estudo estadunidense avaliando o impacto da religião na autoestima sexual das mulheres, os resultados sugeriram que há uma correlação negativa entre elevada religiosidade (em sua maioria Católica) e autoestima sexual (Abbott, Harris, \& Mollen, 2016), relacionando sensações de culpa, devido a uma percepção de que Deus avalia a maior parte das práticas sexuais de maneira negativa.

\section{Bem-estar geral e autoestima}

O self-sexual mostra-se relacionado à satisfação com a vida, ao bem-estar geral e sexual, assim como à autoestima, todos indicadores de qualidade de vida (Hewitt-Stubbs et al., 2016;
Horne \& Zimmer-Gembeck, 2006; Mastro \& Zimmer-Gembeck, 2015; Zimmer-Gembeck, Ducat \& Boislard-Pepin, 2011; Zimmer-Gembeck, 2013; Zimmer-Gembeck \& French, 2016). Estudar o self-sexual pode, além de enriquecer a compreensão da sexualidade, proporcionar o conhecimento necessário para aumentar a qualidade de vida. Cabe enfatizar que possuir um self-sexual saudável vai muito além da esfera da sexualidade. Pensar sexualidade sob a ótica da saúde geral implica em considerar todos os aspectos multidimensionais da sexualidade reconhecidos pela Organização Mundial da Saúde (WHO, 2004). No entanto, trata-se de um conceito novo, não explorado no Brasil, fato que foi corroborado pela carência de estudos que enfoquem sexualidade por intermédio deste paradigma.

Sendo assim, partindo desta breve introdução, salienta-se a importância de estudar os componentes do self-sexual na realidade brasileira. O presente estudo objetiva investigar os componentes do self-sexual (autoestima corporal-sexual, sensação de direito a receber prazer sexual de si mesmo(a), sensação de direito a receber prazer sexual do(a) parceiro(a), autoeficácia para alcançar o prazer sexual, autorreflexão sexual) na população de jovens adultos (18 a 30 anos) brasileiros e as suas associações com a autoestima e satisfação com a vida.

\section{Método}

A presente pesquisa apresenta delineamento quantitativo, descritivo e transversal.

\section{Participantes}

Participaram desse estudo 1380 residentes brasileiros de 18 a 30 anos de idade. Eles foram divididos em seis subgrupos: mulheres heterossexuais (MulheresHéteras) $(\mathrm{n}=866)$; mulheres homossexuais (MulheresHomo) $(\mathrm{n}=36)$; mulheres bissexuais (MulheresBi) $(\mathrm{n}=174)$; homens heterossexuais 
(HomensHéteros) $(\mathrm{n}=227)$; homens homossexuais (HomensHomo) $(\mathrm{n}=42)$ e homens bissexuais (HomensBi) $(\mathrm{n}=22)$. Os indivíduos de orientações sexuais não-binárias, assim como todos que não se identificaram como cisgênero, foram excluídos de qualquer comparação ou correlação devido à baixa representatividade na amostra (menos de 1\%). $53.8 \%$ se identificaram como estudantes e $42.5 \%$ possuíam o Ensino Superior em andamento. 50.3\% dos participantes responderam possuir alguma religião e $30.7 \%$ se declararam como cristãos, católicos e/ou protestantes. $96.7 \%$ relataram já terem tido uma experiência sexual. O sujeito não ter experienciado uma relação sexual com o outro não o desqualifica de ter seu self-sexual analisado, posto que se refere a um constructo pessoal introspectivo, contemplando sensações e percepções dele próprio enquanto agente/objeto sexual dentro da sociedade.

\section{Instrumentos}

\section{Questionário de dados sociodemográficos elaborado para o presente estudo}

O questionário consistia de 21 perguntas para definição das seguintes variáveis: idade, localização, identidade de gênero, cor-de-pele, ocupação, profissão, com quem reside, renda individual e familiar, escolaridade própria e dos pais, orientação sexual, experiência passada e presente de relações sexuais (práticas sexuais acompanhadas) e de relacionamentos amorosos estáveis.

\section{Inventário de Self-Sexual (ISS)}

A tradução que foi concebida para este estudo foi baseada no Male Sexual Subjectivity Inventory - MSSI (Zimmer-Gembeck \& French, 2016). Para o presente estudo, foi realizada uma tradução e adaptação linguística. A adaptação do questionário para a língua portuguesa foi realizada utilizando tradução reversa. O processo de tradução foi realizado por cinco psicólogos fluentes em português e inglês. As perguntas do questionário foram então retrotraduzidas para o inglês por dois psicólogos bilíngues com histórico de residência em países anglófonos. No que tange à análise de conteúdo e análise aparente, o questionário traduzido foi aplicado em 10 adultos jovens universitários, a fim de verificar o entendimento, a adequação dos itens e conteúdos e a formatação das questões. Sendo assim, nos itens em que houve possibilidade de substituição por expressões que melhor descrevessem o conteúdo, adaptações foram realizadas. A escala se propõe a avaliar a subjetividade sexual com foco em 5 componentes fundamentais: autoestima corporal-sexual, sensação de direito a receber prazer sexual de si mesmo(a), sensação de direito a receber prazer sexual do(a) parceiro(a), autoeficácia para alcançar o prazer sexual e autorreflexão sexual. O Iss é composto de 20 itens, com respostas variando de 1 (discordo totalmente) a 5 (concordo totalmente). Após a inversão das perguntas feitas na forma negativa, os resultados indicam que, quanto maior a pontuação, maior a sensação de atitude e apropriação da própria sexualidade. Avaliando os coeficientes de Alpha Cronbach da escala que deu origem, a MSSI (Zimmer-Gembeck \& French, 2016) encontrou 0.78 para autoestima corporal-sexual, 0.83 para sensação de direito a receber prazer sexual de si mesmo(a), 0.72 para sensação de direito a receber prazer sexual do(a) parceiro(a), 0.76 para autoeficácia para alcançar o prazer sexual e 0.73 para autorreflexão sexual, apresentando adequada coerência interna. Para a presente pesquisa, verificaram-se os seguintes Alphas: 0.63 para autoestima corporal-sexual, 0.80 para sensação de direito a receber prazer sexual de si mesmo(a), 0.66 para sensação de direito a receber prazer sexual do(a) parceiro(a), 0.82 para autoeficácia para alcançar o prazer sexual e 0,73 para autorreflexão sexual.

\section{Satisfação com a Vida}

A Escala de Satisfação com a Vida foi concebida por Diener, Emmons, Larsen e Griffin (1985) e foi 
traduzida e adaptada para a população brasileira por Gouveia, Milfont, da Fonseca e de Miranda Coelho (2009). De maneira autoperceptiva, a escala tem como objetivo que o sujeito avalie sua satisfação geral com a vida. Ela contém 5 itens (exemplo: "Em muitas maneiras, minha vida se aproxima aos meus ideais") com respostas variando de 1 (totalmente em desacordo) a 7 (totalmente de acordo). Resultados elevados indicam uma elevada satisfação geral com a vida. Gouveia et al. (2009) encontraram Alpha Cronbach de 0.81 e Silva, do Céu Taveira, Marques e Gouveia (2015) encontraram 0.80 em seu estudo com adolescentes e jovens adultos em Portugal. Para a presente pesquisa, foi encontrado Alpha Cronbach de 0.85 .

\section{Escala de Autoestima de Rosenberg}

A Escala de Autoestima de Rosenberg foi traduzida e adaptada para aplicação na população brasileira por Dini, Quaresma e Ferreira (2004) e revisada por Hutz e Zanon (2011). A escala possui 10 itens, com respostas variando de 1 (discordo totalmente) a 4 (concordo totalmente) e avalia a autoestima dos participantes. Resultados elevados indicam uma elevada autoestima. Um exemplo de item seria: "Eu sinto que possuo diversas qualidades positivas". Em múltiplos estudos apresentou-se elevada confiabilidade durante sua aplicação, encontrando Alpha Cronbach elevados, como 0.89 (Horne \& Zimmer-Gembeck, 2006), 0.90 (Hutz \& Zanon, 2011) e 0.88 (Durso, Latner, \& Ciao, 2016). Para a presente pesquisa, foi encontrado Alpha Cronbach de 0.90 .

\section{Índice de Religiosidade}

A primeira pergunta referente à religiosidade feita aos participantes foi "você possui religiosidade?". Em caso de resposta afirmativa, surgia um campo aberto para especificar qual religião. Independentemente da resposta, a seguir era oferecido o Índice de Religiosidade da Universidade de Duke (P-DUREL). O P-DUREL (Koenig, Parkerson \& Meador, 1997) foi adaptado para aplicação na população brasileira por Moreira-Almeida, Peres, Aloe, Lotufo Neto e Koenig (2008). A escala possui 5 itens de múltipla escolha e se propõe a avaliar as dimensões da religiosidade em amostras brasileiras com características sociodemográficas diversas. Um exemplo de item seria: "As minhas crenças religiosas estão realmente por trás de toda a minha maneira de viver". O P-DUREL teve adequada consistência interna (Alpha Cronbach $>0,80$ ) e confiabilidade teste-reteste (Coeficiente de Correlação Intraclasse $>0,90$ ) em duas amostras distintas investigadas por Taunay et al. (2012). Para a presente pesquisa, foi encontrado Alpha Cronbach de 0.90 .

\section{Procedimentos de coleta de dados e considerações éticas}

A pesquisa foi estruturada na plataforma Survey Monkey e divulgada na rede social Facebook. A chamada foi compartilhada por diversos participantes que repassaram o link a seus contatos após responderem, de forma que mais sujeitos viessem a contribuir com a pesquisa. A plataforma permaneceu disponível para acesso pelo período de 30 dias. 1988 participantes iniciaram a pesquisa, mas 608 não responderam todas as questões. O tempo médio de preenchimento foi de 9 minutos.

Os participantes não receberam dinheiro ou qualquer outra bonificação em troca da sua contribuição na pesquisa. O Comitê de Ética e Pesquisa da Universidade Federal de Ciências da Saúde de Porto Alegre aprovou esta pesquisa (CAAE 67815617.8.0000.5345). Os sujeitos que concordaram em participar da pesquisa assinalaram que estavam de acordo com o termo de consentimento livre e esclarecido na primeira página da pesquisa. Na divulgação dos resultados deste estudo, o nome de nenhum participante é citado. A participação na pesquisa não acarretou nenhuma complicação legal e nenhum dos inventários respondidos ofereceu riscos à dignidade dos participantes. 
De fato, nenhum participante relatou a percepção de algum dano ou desconforto psicológico comprovadamente derivado da participação na pesquisa (durante ou após o encerramento/interrupção do preenchimento da mesma). Por se tratar de uma pesquisa voluntária e isenta de qualquer custo, não houve qualquer tipo de despesa para o participante. Não houve relato de qualquer prejuízo para quem se recusou a participar ou abandonou a pesquisa em qualquer momento do preenchimento do questionário. Embora a participação nesta pesquisa não proporcione nenhum benefício direto ao participante, espera-se que sua contribuição tenha auxiliado na reflexão sobre o tema, assim como na ampliação do estudo do self-sexual.

\section{Análise de dados}

As variáveis quantitativas foram descritas por média e desvio padrão; as variáveis categóricas, como frequências simples (n) e relativas (\%). O teste de correlação de Spearman foi utilizado para avaliar relação linear entre variáveis quantitativas. Para verificar diferença de médias entre os seis subgrupos de gênero e orientação sexual, foi utilizada Análise de variância (ANOVA), seguida pelo teste de Bonferroni para comparações múltiplas, quando necessário. Para avaliar associação entre variáveis categóricas foi utilizado o teste qui-quadrado. $\mathrm{Na}$ sequência foi realizada análise de resíduos para identificar associações com significância estatística entre as categorias das variáveis. Para todas as análises, foi considerado um nível de significância de $5 \%(\mathrm{p}<0,05)$. As análises estatísticas foram realizadas no programa Statistical Package for the Social Sciences (SPSS), versão 23.0 (SPSS INC., IBM Company, Chicago, EUA).

\section{Resultados}

Todas as médias e desvios padrões referentes às variáveis do estudo estão listados nas tabelas 1.1, 1.2 e 2. Diferenças entre indivíduos de identidades de gênero e orientações sexuais distintas foram encontradas em todas variáveis sociodemográficas, assim como nas variáveis frequência de prática masturbatória individual, frequência de relações sexuais, índice de religiosidade, autorreflexão sexual, sensação de direito a receber prazer sexual de si mesmo(a), sensação de direito a receber prazer sexual do(a) parceiro(a), na autoestima geral e no índice de religiosidade.

A comparação das médias e desvios padrões dos homens e mulheres, de acordo com o Inventário de Self-sexual, está descrita na tabela 3. Dentre os componentes do self-sexual, os únicos que apresentaram diferença significativa entre os dois gêneros foram "sensação de direito a receber prazer sexual de si mesmo(a)" e "sensação de direito a receber prazer sexual do(a) parceiro(a)", ambos tendo as mulheres com médias superiores às dos homens.

No que tange às análises correlacionais (tabela 4), todas as subescalas do ISS (autoestima corporal-sexual, sensação de direito a receber prazer sexual de si mesmo(a), sensação de direito a receber prazer sexual do(a) parceiro(a), autoeficácia para alcançar o prazer sexual e autorreflexão sexual) apresentaram correlação significativa com a Escala de Satisfação com a Vida e com a Escala de Auto Estima de Rosenberg. O índice de religiosidade apresentou correlação positiva com a subescala de autoestima corporal-sexual e negativa com as escalas de sensação de direito a receber prazer sexual de si mesmo(a) e sensação de direito a receber prazer sexual do(a) parceiro(a). As demais subescalas de self-sexual (autorreflexão sexual e autoeficácia para alcançar o prazer sexual) não exibiram correlação significativa com o índice de religiosidade.

Quanto à frequência de masturbação (considerando apenas práticas solitárias, sem outras pessoas envolvidas), a frequência mais usual foi a de uma a duas vezes por semana (385 pessoas, $27.9 \%$ ), mas vale ressaltar as $92(6.7 \%)$ que o fazem todos os dias e as $83(6 \%)$ que nunca se masturbaram. 


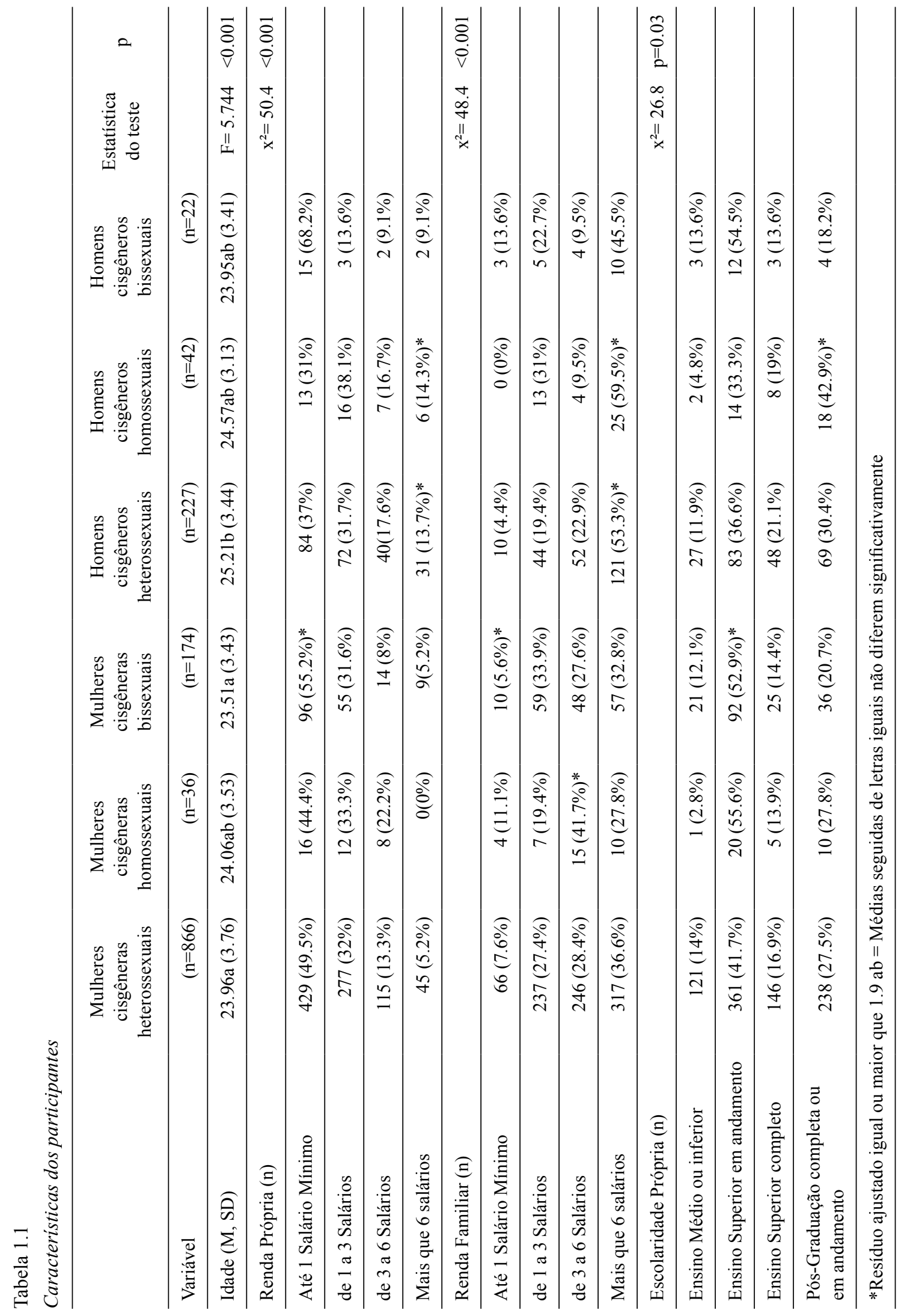


Tabela 1.2

Características dos participantes

\begin{tabular}{|c|c|c|c|c|c|c|c|c|}
\hline & $\begin{array}{c}\text { Mulheres } \\
\text { cisgêneras } \\
\text { heterossexuais }\end{array}$ & $\begin{array}{c}\text { Mulheres } \\
\text { cisgêneras } \\
\text { homossexuais }\end{array}$ & $\begin{array}{l}\text { Mulheres } \\
\text { cisgêneras } \\
\text { bissexuais }\end{array}$ & $\begin{array}{c}\text { Homens } \\
\text { cisgêneros } \\
\text { heterossexuais }\end{array}$ & $\begin{array}{c}\text { Homens } \\
\text { cisgêneros } \\
\text { homossexuais }\end{array}$ & $\begin{array}{l}\text { Homens } \\
\text { cisgêneros } \\
\text { bissexuais }\end{array}$ & $\begin{array}{c}\text { Estatística } \\
\text { do teste }\end{array}$ & $\mathrm{p}$ \\
\hline $\begin{array}{l}\text { Relacionamento } \\
\text { Estável (n) }\end{array}$ & & & & & & & $\mathrm{x}^{2}=35.5$ & $\mathrm{p}=0.002$ \\
\hline $\begin{array}{l}\text { Solteiro(a) sem } \\
\text { experiência de } \\
\text { relacionamentos } \\
\text { duradouros }\end{array}$ & $77(8.9 \%)$ & $1(2.8 \%)$ & $12(6.9 \%)$ & $22(9.7 \%)$ & $8(19 \%)$ & $6(27.3 \%)$ & & \\
\hline $\begin{array}{l}\text { Solteiro(a) com } \\
\text { experiência de } \\
\text { relacionamentos } \\
\text { duradouros }\end{array}$ & $213(24.6 \%)$ & $11(30.6 \%)$ & $\begin{array}{r}62 \\
(35.6 \%)^{*}\end{array}$ & $57(25.1 \%)$ & $15(35.7 \%)$ & $8(36.4 \%)$ & & \\
\hline $\begin{array}{l}\text { Em um } \\
\text { relacionamento } \\
\text { curto }\end{array}$ & $104(12 \%)$ & $3(8.3 \%)$ & $\begin{array}{r}19 \\
(10.9 \%)\end{array}$ & $18(7.9 \%)$ & $5(11.9 \%)$ & $1(4.5 \%)$ & & \\
\hline $\begin{array}{l}\text { Em um } \\
\text { relacionamento } \\
\text { com mais de } 6 \\
\text { meses }\end{array}$ & $472(54.5 \%)$ & $21(58.3 \%)$ & $\begin{array}{r}81 \\
(47.6 \%)\end{array}$ & $130(57.3 \%)$ & $14(33.3 \%)^{*}$ & $7(31.8 \%)$ & & \\
\hline
\end{tabular}

Frequência

de Prática

Masturbatória

Indiv. (n)

\begin{tabular}{|c|c|c|c|c|c|c|}
\hline Todos os dias & $22(2.5 \%)$ & $1(2.8 \%)$ & $15(8.6 \%)$ & $34(15 \%)^{*}$ & $13(31 \%)^{*}$ & $\begin{array}{r}5 \\
22.7 \%)^{*}\end{array}$ \\
\hline $\begin{array}{l}\text { De } 3 \text { a } 4 \text { vezes } p / \\
\text { semana }\end{array}$ & $123(14.2 \%)$ & $5(13.9 \%)$ & $\begin{array}{r}38 \\
(21.8 \%)\end{array}$ & $83(36.6 \%)^{*}$ & $17(40.5 \%)^{*}$ & $\begin{array}{r}9 \\
(40.9 \%)^{*}\end{array}$ \\
\hline $\begin{array}{l}\text { De } 1 \text { a } 2 \text { vezes } p / \\
\text { semana }\end{array}$ & $222(25.6 \%)$ & $16(44.4 \%)^{*}$ & $\begin{array}{r}69 \\
(39.7 \%)^{*}\end{array}$ & $62(27.3 \%)$ & $6(14.3 \%)$ & $6(27.3 \%)$ \\
\hline $\begin{array}{l}\text { Uma vez a cada } \\
\text { duas semanas }\end{array}$ & $\begin{array}{r}163 \\
(18.8 \%)^{*}\end{array}$ & $5(13.9 \%)$ & $\begin{array}{r}25 \\
(14.4 \%)\end{array}$ & $21(9.3 \%)$ & $4(9.5 \%)$ & $1(4.5 \%)$ \\
\hline Uma vez por mês & $\begin{array}{r}110 \\
(12.7 \%)^{*}\end{array}$ & $3(8.3 \%)$ & $12(6.9 \%)$ & $9(4.0 \%)$ & $1(2.4 \%)$ & $0(0 \%)$ \\
\hline $\begin{array}{l}\text { Semestral (De } 1 \text { a } 3 \\
\text { vezes p/ semestre) }\end{array}$ & $93(10.7 \%)^{*}$ & $5(13.9 \%)$ & $11(6.3 \%)$ & $4(1.8 \%)$ & $0(0 \%)$ & $0(0 \%)$ \\
\hline $\begin{array}{l}1 \mathrm{vez} \text { / ano ou } \\
\text { menos }\end{array}$ & $60(6.9 \%)^{*}$ & $1(2.8 \%)$ & $3(1.7 \%)$ & $6(2.6 \%)$ & $1(2.4 \%)$ & $0(0 \%)$ \\
\hline Nunca praticou & $73(8.4 \%)^{*}$ & $0(0 \%)$ & $1(0.6 \%)$ & $8(3.5 \%)$ & $0(0 \%)$ & $1(4.5 \%)$ \\
\hline
\end{tabular}




\begin{tabular}{|c|c|c|c|c|c|c|c|c|}
\hline & $\begin{array}{c}\text { Mulheres } \\
\text { cisgêneras } \\
\text { heterossexuais }\end{array}$ & $\begin{array}{c}\text { Mulheres } \\
\text { cisgêneras } \\
\text { homossexuais }\end{array}$ & $\begin{array}{c}\text { Mulheres } \\
\text { cisgêneras } \\
\text { bissexuais }\end{array}$ & $\begin{array}{c}\text { Homens } \\
\text { cisgêneros } \\
\text { heterossexuais }\end{array}$ & $\begin{array}{c}\text { Homens } \\
\text { cisgêneros } \\
\text { homossexuais }\end{array}$ & $\begin{array}{c}\text { Homens } \\
\text { cisgêneros } \\
\text { bissexuais }\end{array}$ & $\begin{array}{l}\text { Estatística } \\
\text { do teste }\end{array}$ & $\mathrm{p}$ \\
\hline $\begin{array}{l}\text { Frequência de } \\
\text { Prática Sexual (n) }\end{array}$ & & & & & & & $\mathrm{x}^{2}=39.2$ & $<0.001$ \\
\hline Todos os dias & $16(1.8 \%)^{*}$ & $0(\%)$ & $1(0.6 \%)$ & $0(0 \%)$ & $2(4.8 \%)^{*}$ & $0(\%)$ & & \\
\hline $\begin{array}{l}\text { De } 3 \text { a } 4 \text { vezes } p / \\
\text { semana }\end{array}$ & $167(19.3 \%)$ & $8(22.2 \%)$ & $\begin{array}{r}32 \\
(18.4 \%)\end{array}$ & $41(18.1 \%)$ & $3(7.1 \%)$ & $5(22.7 \%)$ & & \\
\hline $\begin{array}{l}\text { De } 1 \text { a } 2 \text { vezes p/ } \\
\text { semana }\end{array}$ & $327(37.8 \%)$ & $14(38.9 \%)$ & $\begin{array}{r}75 \\
(43.1 \%)\end{array}$ & $97(42.7 \%)$ & $13(31 \%)$ & $4(18.2 \%)$ & & \\
\hline $\begin{array}{l}\text { Uma vez a cada } \\
\text { duas semanas }\end{array}$ & $118(13.6 \%)$ & $5(13.9 \%)$ & $\begin{array}{r}24 \\
(13.8 \%)\end{array}$ & $36(15.9 \%)$ & $9(21.4 \%)$ & $5(22.7 \%)$ & & \\
\hline Uma vez por mês & $82(9.5 \%)$ & $2(5.6 \%)$ & $14(8 \%)$ & $18(7.9 \%)$ & $7(16.7 \%)$ & $3(13.6 \%)$ & & \\
\hline $\begin{array}{l}\text { Semestral (De } 1 \text { a } 3 \\
\text { vezes p/ semestre) }\end{array}$ & $82(9.5 \%)$ & $4(11.1 \%)$ & $17(9.8 \%)$ & $15(6.6 \%)$ & $7(16.7 \%)$ & $2(9.1 \%)$ & & \\
\hline $\begin{array}{l}1 \text { vez } \mathrm{p} / \text { ano ou } \\
\text { menos }\end{array}$ & $37(4.3 \%)$ & $2(5.6 \%)$ & $7(4 \%)$ & $10(4.4 \%)$ & $1(2.4 \%)$ & $\begin{array}{r}3 \\
(13.6 \%)^{*}\end{array}$ & & \\
\hline Nunca praticou & $37(4.3 \%)$ & $1(2.8 \%)$ & $4(2.3 \%)$ & $10(4.4 \%)$ & $0(0 \%)$ & $0(0 \%)$ & & \\
\hline Religião (n) & & & & & & & $\mathrm{x}^{2}=114.1$ & $<0.001$ \\
\hline Ateu / Agnóstico & $349(40.3 \%)$ & $26(72.2 \%)^{*}$ & $\begin{array}{r}119 \\
(68.4 \%)^{*}\end{array}$ & $\begin{array}{r}142 \\
(62.6 \%)^{*}\end{array}$ & $32(76.2 \%)^{*}$ & $\begin{array}{r}15 \\
(68.2 \%)\end{array}$ & & \\
\hline $\begin{array}{l}\text { Cristã / Católica / } \\
\text { Protest. }\end{array}$ & $\begin{array}{r}335 \\
(38.7 \%)^{*}\end{array}$ & $4(11.1 \%)$ & $\begin{array}{r}20 \\
(11.5 \%)\end{array}$ & $54(23.8 \%)$ & $3(7.1 \%)$ & $4(18.2 \%)$ & & \\
\hline Espírita & $67(7.7 \%)$ & $3(8.3 \%)$ & $16(9.2 \%)$ & $8(3.5 \%)$ & $2(4.8 \%)$ & $0(0 \%)$ & & \\
\hline Evangélica & $55(6.4 \%)$ & $2(5.6 \%)$ & $6(3.4 \%)$ & $11(4.8 \%)$ & $2(4.8 \%)$ & $2(9.1 \%)$ & & \\
\hline Outra Religião & $60(6.9 \%)$ & $1(2.8 \%)$ & $13(7.5 \%)$ & $12(5.3 \%)$ & $3(7.1 \%)$ & $1(4.5 \%)$ & & \\
\hline
\end{tabular}


Tabela 2.

Subescalas de self-sexual, escala de satisfação com a vida, autoestima geral e índice de religiosidade dentre os 6 subgrupos de identidade de gênero e orientação sexual

\begin{tabular}{|c|c|c|c|c|c|c|c|c|}
\hline & $\begin{array}{c}\text { Mulheres } \\
\text { cisgêneras } \\
\text { heterossexuais }\end{array}$ & $\begin{array}{l}\text { Mulheres } \\
\text { cisgêneras } \\
\text { homossexuais }\end{array}$ & $\begin{array}{l}\text { Mulheres } \\
\text { cisgêneras } \\
\text { bissexuais }\end{array}$ & $\begin{array}{c}\text { Homens } \\
\text { cisgêneros } \\
\text { heterossexuais }\end{array}$ & $\begin{array}{l}\text { Homens } \\
\text { cisgêneros } \\
\text { homossexuais }\end{array}$ & $\begin{array}{l}\text { Homens } \\
\text { cisgêneros } \\
\text { bissexuais }\end{array}$ & $\mathrm{F}$ & Valor $\mathrm{p}$ \\
\hline & Escalas & $(\mathrm{n}=866)$ & $(n=36)$ & $(\mathrm{n}=174)$ & $(n=227)$ & $(n=42)$ & $(n=22)$ & \\
\hline $\begin{array}{l}\text { Autoestima corporal- } \\
\text { sexual }\end{array}$ & $11.49(2.88)$ & $11.13(2.88)$ & $\begin{array}{l}11.64 \\
(3.02)\end{array}$ & $11.63(2.71)$ & $10.92(3.18)$ & $\begin{array}{l}10.36 \\
(2.17)\end{array}$ & 1.311 & 0.256 \\
\hline Autorreflexão sexual & $15.73^{\mathrm{a}}(2.72)$ & $\begin{array}{r}16.25^{\mathrm{ab}} \\
(2.47)\end{array}$ & $\begin{array}{l}16.69^{\mathrm{b}} \\
(2.48)\end{array}$ & $15.41^{\mathrm{a}}(2.84)$ & $\begin{array}{r}15.64^{\mathrm{ab}} \\
(2.93)\end{array}$ & $\begin{array}{r}16.18^{\mathrm{ab}} \\
(2.92)\end{array}$ & 5.063 & $<0.001^{*}$ \\
\hline $\begin{array}{l}\text { Sensação de direito a } \\
\text { receber prazer sexual } \\
\text { de si mesmo(a) }\end{array}$ & $\begin{array}{r}16.77^{\mathrm{ac}} \\
(2.65)\end{array}$ & $\begin{array}{r}17.75^{\mathrm{ab}} \\
(2.03)\end{array}$ & $\begin{array}{l}18.24^{\mathrm{b}} \\
(2.13)\end{array}$ & $16.26^{\mathrm{c}}(2.71)$ & $\begin{array}{r}17.26^{\mathrm{abc}} \\
(2.81)\end{array}$ & $\begin{array}{r}16.54^{\mathrm{abc}} \\
(2.48)\end{array}$ & 14.908 & $<0.001^{*}$ \\
\hline $\begin{array}{l}\text { Sensação de direito a } \\
\text { receber prazer sexual } \\
\text { do(a) parceiro(a) }\end{array}$ & $17.84^{\mathrm{a}}(1.84)$ & $\begin{array}{r}18.02^{\mathrm{ab}} \\
(1.50)\end{array}$ & $\begin{array}{l}18.43^{\mathrm{b}} \\
(1.52)\end{array}$ & $16.92^{\mathrm{c}}(2.15)$ & $\begin{array}{l}17.38^{\mathrm{ac}} \\
(2.24)^{-}\end{array}$ & $\begin{array}{r}16.90^{\mathrm{ac}} \\
(2.50)\end{array}$ & 12.969 & $<0.001^{*}$ \\
\hline $\begin{array}{l}\text { Autoeficácia para } \\
\text { alcançar o prazer } \\
\text { sexual }\end{array}$ & $15.86(2.86)$ & $16.08(2.67)$ & $\begin{array}{l}16.44 \\
(2.48)\end{array}$ & $15.63(2.67)$ & $15.85(2.46)$ & $\begin{array}{l}16.45 \\
(2.72)\end{array}$ & 1.987 & 0.077 \\
\hline $\begin{array}{l}\text { Satisfação com a } \\
\text { Vida }\end{array}$ & $22.75(6.44)$ & $21.72(6.13)$ & $\begin{array}{l}22.72 \\
(6.13)\end{array}$ & $22.48(6.50)$ & $22.40(7.18)$ & $\begin{array}{l}20.09 \\
(4.59)\end{array}$ & 1.147 & 0.333 \\
\hline Autoestima Geral & $19.58^{\mathrm{a}}(5.65)$ & $\begin{array}{r}18.77^{\mathrm{ab}} \\
(5.78)\end{array}$ & $\begin{array}{l}17.95^{\mathrm{b}} \\
(6.05)\end{array}$ & $20.39^{\mathrm{a}}(5.51)$ & $\begin{array}{c}19.61^{\mathrm{ab}} \\
(6.34)\end{array}$ & $\begin{array}{c}18.86^{\mathrm{ab}} \\
(3.58)\end{array}$ & 3.900 & 0.001 \\
\hline $\begin{array}{l}\text { Índice de } \\
\text { Religiosidade }\end{array}$ & $14.06^{\mathrm{a}}(6.20)$ & $9.02^{\mathrm{b}}(4.46)$ & $\begin{array}{l}10.80^{\mathrm{b}} \\
(5.83)\end{array}$ & $10.88^{b}(6.24)$ & $8.35^{\mathrm{b}}(5.10)$ & $\begin{array}{l}10.13^{\mathrm{b}} \\
(5.06)\end{array}$ & 30.064 & $<0.001^{*}$ \\
\hline \multicolumn{9}{|c|}{$\mathrm{abc}=$ Médias seguidas de letras iguais não diferem significativamente pelo teste post-hoc de Bonferroni } \\
\hline${ }^{*} \mathrm{p}<0.05$ & & & & & & & & \\
\hline
\end{tabular}


Tabela 3.

Subescalas de self-sexual, escala de satisfação com a vida, autoestima geral e índice de religiosidade comparando homens e mulheres cisgêneros

\begin{tabular}{lcccc}
\hline & $\begin{array}{c}\text { Mulheres } \\
\text { cisgêneras }\end{array}$ & $\begin{array}{c}\text { Homens } \\
\text { Cisgêneros }\end{array}$ & F & Valor $p$ \\
\hline Variável (M, SD) & $(\mathrm{n}=1078)$ & $(\mathrm{n}=291)$ & \\
\hline Autoestima Corporal-Sexual & $11.51(2.90)$ & $11.4(2.77)$ & 1.597 & 0.677 \\
\hline Autorreflexão Sexual & $15.90(2.70)$ & $15.50(2.86)$ & 1.367 & 0.35 \\
\hline Sensação de direito a receber prazer sexual de si mesmo(a) & $17.04(2.61)$ & $16.43(2.72)$ & 0.032 & 0.001 \\
\hline Sensação de direito a receber prazer sexual do(a) parceiro(a) & $17.94(1.79)$ & 16.99 & 11.45 & $<0.001$ \\
\hline Autoeficácia para alcançar o prazer sexual & $15.97(2.80)$ & $15.73(2.64)$ & 0.189 & 0.176 \\
\hline Satisfação com a Vida & $22.61(6.46)$ & $22.29(6.49)$ & 0.33 & 0.463 \\
\hline Autoestima Geral & $19.28(5.75)$ & $20.1(5.52)$ & 0.84 & 0.17 \\
\hline Índice de Religiosidade & $13.37(6.25)$ & $10.46(6.05)$ & 2.381 & $<0.001$ \\
\hline
\end{tabular}

Tabela 4.

Correlações entre as escalas.

\begin{tabular}{lcccccc}
\hline Escalas de self-sexual & Satisfação c/ a Vida & Autoestima Geral & \multicolumn{2}{c}{ Religiosidade } \\
\hline & $\mathrm{rS}$ & $\mathrm{p}$ & $\mathrm{rS}$ & $\mathrm{p}$ & $\mathrm{rS}$ & $\mathrm{p}$ \\
\hline Autoestima Corporal-Sexual & $.266^{* *}$ & .000 & $.423^{* *}$ & .000 & $.097^{* *}$ & .000 \\
\hline Autorreflexão Sexual & $.086^{* *}$ & .001 & $.142^{* *}$ & .000 & -.049 & .069 \\
\hline $\begin{array}{l}\text { Sensação de direito a receber prazer sexual de si } \\
\text { mesmo(a) }\end{array}$ & $.132^{* *}$ & .000 & $.127^{* *}$ & .000 & $-.226^{* *}$ & .000 \\
\hline $\begin{array}{l}\text { Sensação de direito a receber prazer sexual do(a) } \\
\text { parceiro(a) }\end{array}$ & $.075^{* *}$ & .005 & $.068^{*}$ & .012 & $-.071^{* *}$ & .009 \\
\hline \begin{tabular}{l} 
Autoeficácia para alcançar o prazer sexual \\
\hline
\end{tabular} & $.144^{* *}$ & .000 & $.265^{* *}$ & .000 & .051 & .059 \\
\hline
\end{tabular}

rS: correlação de Spearman; Valor p para teste de correlação de Spearman 
Já em relação às dimensões do self-sexual, comparado aos demais grupos, os HomensHéteros apresentaram os graus mais baixos em três subescalas de self-sexual (autoeficácia para alcançar o prazer sexual, sensação de direito a receber prazer sexual de si mesmo e autorreflexão sexual) além de terem tido o penúltimo maior grau na subescala "sensação de direito a receber prazer sexual do parceiro", apenas a frente dos HomensBi (tabela 2). Por outro lado, os HomensHéteros obtiveram o maior grau (20.39) na escala de Autoestima de Rosenberg. Considerando apenas as diferenças estatisticamente significativas, o grupo de maior destaque é o das Mulheres $B i$ que apresentaram escores maiores que os grupos heterossexuais nas subescalas de "autorreflexão sexual", "direito a receber prazer sexual de si mesmo" e "direito a receber prazer do parceiro". Mesmo assim, a única variação significativa na escala de Autoestima de Rosenberg foi MulheresHéteras com níveis superiores às MulheresBi. Nas subescalas de "autoestima corporal-sexual" e "autoeficácia para receber prazer de si mesmo", nenhum grupo se sobressaiu.

\section{Discussão}

Este estudo buscou mapear o self-sexual de uma parcela importante de jovens adultos brasileiros. Se este componente da sexualidade das pessoas (especialmente jovens) é relevante para o desenvolvimento de uma vida com mais qualidade e saúde, precisamos compreender de que maneira componentes sociais (religião, educação, cultura, etc.) podem estar correlacionados com uma sensação de maior apropriação e atitude sobre seu self-sexual. Afinal, compreender o que está associado a uma maior liberdade, fluidez e plenitude da sexualidade pode ser uma maneira de melhorar a qualidade de vida.

No estudo australiano acerca do constructo de self-sexual (Horne \& Zimmer-Gembeck, 2006) foi identificada uma correlação positiva entre self-sexual e as escalas de satisfação com a vida e a autoestima geral, o que foi corroborado por estudos internacionais realizados posteriormente (Hewitt-Stubbs et al., 2016; Mastro \& Zimmer-Gembeck, 2015; Zimmer-Gembeck et al., 2011; Zimmer-Gembeck, 2013; Zimmer-Gembeck \& French, 2016). Conforme esperado, com instrumentos adaptados para o português brasileiro, avaliando jovens brasileiros, as escalas de self-sexual de fato apresentaram tal correlação com as escalas de autoestima e satisfação com a vida. Esses achados salientam a relevância da educação sexual e da saúde sexual como potencializadores de saúde geral-melhorando até a performance acadêmica (Barr et al., 2014; García-Gómez et al., 2017; Sánchez, 2009).

Dentre todas as subescalas de self-sexual, é compreensível que a escala com maior correlação positiva com a escala de Autoestima Geral fosse a escala de Autoestima Corporal-Sexual. Afinal, ambas visam mensurar processos subjetivos de autoavaliação e autovalorização. A Autoestima Geral contempla uma esfera mais ampla, incluindo o respeito que uma pessoa tem por si mesma, a autoconfiança e visões positivas de si (Abdel-Khalek, 2016), enquanto que a Autoestima Corporal-Sexual foca especificamente a aceitação e valorização do seu próprio corpo enquanto veículo/ferramenta de sua sexualidade (Horne \& Zimmer-Gembeck 2006; Zimmer-Gembeck 2016).

Por outro lado, ao investigar o impacto que a religião poderia ter no desenvolvimento do self-sexual de jovens adultos, este estudo partiu da premissa de que, quanto mais presente fosse a religião na vida da pessoa, menos gestão ela possuiria sobre sua sexualidade. Isto porque, se a religiosidade for incorporada de forma a atribuir culpa a determinados impulsos, desejos e comportamentos sexuais, ao desestimular a exploração do corpo e do prazer pessoal e da pessoa com quem se relaciona, o resultado seria uma sexualidade rígida e carente de sensações de atitude e livre-arbítrio sexual (Ellison, 2011; Garceau \& Ronis, 2017; Woo, Morshedian, Brotto \& Gorzalka, 2012). A 
correlação negativa do índice de religiosidade com as variáveis "sensação de direito a receber prazer sexual de si mesmo(a)" ( $p<0,001)$ e "sensação de direito a receber prazer sexual do(a) parceiro(a)" $(p=0,009)$ confirmaram essa expectativa. Ao descrever essas duas variáveis, Horne e Zimmer-Gembeck (2006) comentam que a habilidade para aceitar e reconhecer seus próprios desejos sexuais é um componente importante para interpretar experiências sexuais e tomar decisões sexuais, habilidade esta que possivelmente tenha sido subdesenvolvida em pessoas cuja religiosidade rígida tenha negligenciado espaços de reflexão e autoaceitação sexual.

Se comparados a uma amostra de jovens australianos, conforme registrado por Zimmer-Gembeck e French (2016), a presente amostra de jovens adultos brasileiros, tanto masculina quanto feminina, apresentou médias superiores em Autorreflexão Sexual, Autoeficácia Sexual, de Sensação de direito a receber prazer sexual de si mesmo(a) e sensação de direito a receber prazer sexual do(a) parceiro(a). Ou seja, todas as dimensões mostraram-se com níveis superiores, exceto autoestima corporal-sexual, na qual os escores australianos superaram os brasileiros. Embora seja difícil executar uma comparação fidedigna entre as amostras devido a limitações metodológicas, os resultados despertam curiosidade. A avaliação de Robinson e Davies (2008) da (falta de) evolução na educação sexual na Austrália aponta que esta ainda deixa a desejar no que diz respeito a uma falta de modernização da visão da sexualidade, reforçando normas sociais conservadoras e tabus religiosos, de modo a promover preconceito e valores heterossexistas. Por outro lado, Heilborn (2009), em seu estudo sobre a sexualidade brasileira, confronta e desmistifica a crença popular de que o Brasil é um país sexualmente desinibido, reportando tendências conservadoras em relação às práticas sexuais e ao que se espera de cada gênero (como os parâmetros de virilidade masculina e castidade feminina). Em um estudo subsequente, Heilborn e
Cabral (2013) encontraram que, embora ainda se percebesse resistência à mudança de perspectiva em múltiplas concepções relacionadas aos papéis de gênero, foi detectada uma maior aceitação de práticas anteriormente consideradas desviantes, $\mathrm{o}$ que indicaria uma "moralidade sexual mais moderna". Tal constatação é condizente com a possível relação de maior apropriação e liberdade sexual indicada pelos escores de self-sexual da presente amostra de brasileiros em comparação a de australianos (Zimmer-Gembeck \& French, 2016).

Outro foco deste estudo visou avaliar as diferenças das variáveis de self-sexual entre diferentes identidades de gênero e orientações sexuais. Os resultados indicaram que homens e mulheres distinguiram significativamente $(\mathrm{p}<0.05)$ somente nas variáveis "sensação de direito a receber prazer sexual de si mesmo(a)" e "sensação de direito a receber prazer sexual do(a) parceiro(a)", ambas tendo as mulheres com escores médios superiores aos homens. Estes resultados diferem de uma das primeiras (e únicas encontradas até o momento da atual pesquisa) comparações entre gêneros, feita por Zimmer-Gembeck e French (2016) na Austrália, em que homens jovens reportaram maior sensação de direito a receberem prazer de si mesmos e autoeficácia para alcançar o prazer sexual, ao mesmo tempo em que ambas as pesquisas encontraram escores semelhantes quanto a mulheres reportarem maior sensação de direito a receber prazer sexual do(a) parceiro(a).

Ao avaliarmos a "sensação de direito a receber prazer de si mesmo(a)", uma primeira associação que pode ser feita é com a prática e frequência de masturbação, bem como a cultura de aceitação dela - ou falta de, nos casos em que tal prática é encarada como tabu. O que dificulta a interpretação dos elevados escores das mulheres é o fato de que elas possuem uma menor frequência masturbatória (inclusive $9 \%$ das participantes jamais o fizeram) e fazem parte, principalmente o subgrupo das mulheres heterossexuais, da maior concentração de cristãs, católicas e protestantes, o que supostamente seria 
um entrave na sensação de permitir-se dar prazer (Ellison, 2011; Garceau \& Ronis, 2017; Woo et al., 2012). No entanto, na presente pesquisa elas afirmaram que se sentem no direito de receber prazer de si. Muito se avançou em termos de equidade de gênero e mulheres jovens se sentem empoderadas em diversos aspectos da vida, incluindo, possivelmente, a consciência do direito de receber prazer (Baumel, 2014). Porém, talvez seja preciso avançar ainda no tabu da masturbação feminina, pondo em prática em termos comportamentais aquilo que a mente conscientemente já aceita (reconhecem suas necessidades sexuais, mas não tem iniciativa para fazer valer seus direitos sexuais). Por outro lado, deve-se contemplar a possibilidade que uma pessoa confortável com a própria sexualidade (e com o direito de se masturbar sem culpa) possa simplesmente optar por raramente se masturbar, como uma preferência pessoal e não como um sintoma de uma educação repressora.

Quanto à "sensação de direito a receber prazer do(a) outro(a)", Galinsky e Sonenstein (2011) relataram existir uma discrepância entre a consistência com que os gêneros atingem o orgasmo: os homens, em 9 a cada 10 interações sexuais, e as mulheres, menos da metade das vezes. Dentre as possíveis justificativas para esta "meta não atingida" estão os valores sociais (não valorizar o prazer da mulher), o desconhecimento anatômico da genitália feminina (da parte de ambos os sexos), a dificuldade inerente de comunicação com novos(as) parceiros(as) durante o sexo, entre outros (Galinsky \& Sonenstein, 2011).

Outra possível e mais completa comparação a ser feita seria entre os seis subgrupos de identidade de gênero e orientação sexual. Diferenças relevantes $(\mathrm{p}<0.05)$ entre médias das subescalas de self-sexual só apareceram na "autorreflexão sexual" e na "sensação de direito a receber prazer sexual", tanto de "si mesmo(a)" quanto "do(a) parceiro(a)". Em todas essas, as mulheres bissexuais se sobressaíram com os maiores escores. Estes dados são condizentes com a noção de que mulheres bissexuais podem ter maior livre arbítrio para vivenciar sua sexualidade sem intervenção de tabus e demais cognições, como indica o estudo de sociosexualidade de Semenyna, Belu, Vasey e Honey (2018). Por outro lado, estes dados contrastam com os maiores índices de depressão, ansiedade e propensão ao suicídio que pessoas bissexuais tendem a sofrer (Marshal et al., 2011; Pompili et al., 2014).

Ainda há muitos valores sociais compostos de preconceitos, estigmas e moralismos afetando a qualidade de vida geral e o bem-estar sexual de jovens adultos. Isto sem falar na população transgênera, não contemplada neste estudo, cujo índices de homicídio são maiores no Brasil do que em qualquer outro lugar do mundo. Enquanto tais valores sociais mantiverem suas características, seguirá havendo prejuízo e sofrimento no desenvolvimento da vida sexual das pessoas, principalmente para as minorias sexuais. Quando Horne e Zimmer-Gembeck (2006) conceberam o primeiro inventário de self- sexual (específico para mulheres), a preocupação subjacente era de mapear de que maneira as jovens mulheres estavam sendo objetificadas e privadas de um desenvolvimento de um self-sexual mais livre e saudável. Uma década depois, ao comparem homens e mulheres (Zimmer-Gembeck \& French, 2016), a discrepância de resultados entre os gêneros indicava vestígios de valores sociais que não favoreciam o desenvolvimento sexual das mulheres. O presente estudo encontra um panorama diferente na população brasileira.

Ao contrário do previsto em uma sociedade que ainda sujeita as mulheres a elevados níveis de objetificação, na amostra deste estudo, as mulheres não obtiveram resultados menores que os homens no inventário de self-sexual, apresentando inclusive níveis maiores nas subescalas de sensação de direito a receber prazer sexual de si mesmo(a) e sensação de direito a receber prazer sexual do parceiro(a). Assim, encontramos, de forma surpreendente, que, apesar de ainda imperarem os 
valores patriarcais na cultura brasileira, algumas mudanças têm ocorrido de forma a promover menos restrições morais à sexualidade feminina. Quando comparações foram feitas incluindo diferentes orientações sexuais, as mulheres bissexuais se destacaram com pontuações maiores em três subescalas, apesar de possuírem a menor autoestima geral e serem sabidamente um grupo de risco para maiores níveis de depressão, ansiedade e ideação suicida. Esse achado incentiva que mais estudos visem compreender de que forma o desenvolvimento do self-sexual das mulheres bissexuais se diferencia dos demais.

Ao investigar o impacto que a religiosidade (em sua maioria cristã) possuía no self-sexual, alguns resultados corroboraram a premissa de que uma cultura que reprime a a autoexploração do próprio corpo, proporciona menores níveis de sensação de direito a receber prazer de si e do outro. No entanto, é recomendável levantar mais considerações a respeito da (i)moralidade da sexualidade enquanto tabu no Brasil nos tempos atuais, que contemplem — mas não se limitem — à religiosidade.

\section{Conclusão}

Conforme esperado, o self-sexual apresentou uma relação significativa com a satisfação com a vida e a autoestima geral. Deste modo, reforça-se a necessidade de compreender melhor tal constructo, assim como a de promover uma educação sexual que propicie que os jovens desenvolvam gerência sobre sua própria sexualidade - e não sejam objetos moldados para atenderem expectativas externas.

Durante o recrutamento dos participantes deste estudo, chamou atenção a discrepância do comportamento entre gêneros: enquanto as mulheres estavam rapidamente aderindo à pesquisa, os homens surgiam em números drasticamente menores, o que por si só leva ao questionamento: por que os homens possuem menos interesse em compartilhar suas percepções de sexualidade? De que maneira a cultura e (a falta de) educação sexual podem estar contribuindo para tal comportamento? Além disso, através do método de coleta, não foi possível acessar a população transgênera ou de outras identificações não-binárias (menos de 1\% da amostra se identificou desta forma), deixando como meta que novos estudos de self-sexual busquem representar essa parcela da população, para enriquecer a compreensão de como o self-sexual se aplica a essas pessoas e como se mostra neste país.

O presente estudo reitera a concepção de que o self-sexual possui uma correlação significativa com a satisfação com a vida e a autoestima geral das pessoas, reforçando a relevância de multiplicar e aprofundar pesquisas neste campo. Considerando as limitações desse estudo, outras variáveis podem ser avaliadas, com amostras mais variadas de um número maior de regiões do Brasil e de outros países. $\mathrm{O}$ aperfeiçoamento do conhecimento de como se comporta o self-sexual e a maneira que se pode aumentar a sensação de gerência sobre a própria sexualidade é fundamental para a construção de um desenvolvimento mais saudável, o que pode e deve ser aplicado nas intervenções nas escolas, nas práticas clínicas e na cultura em geral.

\section{Referências}

Abbott, D. M., Harris, J. E., \& Mollen, D. (2016). The impact of religious commitment on women's sexual self-esteem. Sexuality \& Culture, 20(4), 10631082. https://doi.org/10.1007/s12119-016-9374-x

Abdel-Khalek, A. M. (2016). Introduction to the psychology of self-esteem. Em F. Holloway (Ed.), Self-esteem: Perspectives, influences, and improvement strategies (pp. 1-23). Nueva York, NY: Nova Science Publisher.

Amos, N., \& McCabe, M. (2016). Positive perceptions of genital appearance and feeling sexually attractive: Is it a matter of sexual esteem? $A r$ chives of Sexual Behavior, 45(5), 1249-1258. https://doi.org/10.1007/s10508-015-0680-4 
Barr, E. M., Goldfarb, E. S., Russell, S., Seabert, D., Wallen, M., \& Wilson, K. L. (2014). Improving sexuality education: The development of teacher-preparation standards. Journal of School Health, 84(6), 396-415. https://doi.org/10.1111/ josh. 12156

Baumel, S. W. (2014). Investigando o papel da masturbação na sexualidade da mulher (Dissertação de mestrado). Recuperado de http://repositorio. ufes.br/handle/10/3094

Diener, E. D., Emmons, R. A., Larsen, R. J., \& Griffin, S. (1985). The satisfaction with life scale. Journal of personality assessment, 49(1), 71-75. https://doi.org/10.1207/s15327752jpa4901_13

Dini, G., Quaresma, M., \& Ferreira, L. (2004). Adaptação cultural e validação da versão brasileira da escala de auto-estima de Rosenberg. Revista Brasileira de Cirurgia Plástica, 19(1), 41-52.

Durso, L. E., Latner, J. D., \& Ciao, A. C. (2016). Weight bias internalization in treatment-seeking overweight adults: Psychometric validation and associations with self-esteem, body image, and mood symptoms. Eating Behaviors, 21, 104-108. https://doi.org/10.1016/j.eatbeh.2016.01.011

Ellis, B. J. (2004). Timing of pubertal maturation in girls: An integrated life history approach. Psychological Bulletin, 130(6), 920-958.

Ellison, D. M. (2011). Religious negativism and fantasy guilt. The Family Journal, 19(1), 101-107. https://doi.org/10.1177/1066480710388754

Galinsky, A. M., \& Sonenstein, F. L. (2011). The association between developmental assets and sexual enjoyment among emerging adults. Journal of Adolescent Health, 48(6), 610-615. https:// doi.org/10.1016/j.jadohealth.2010.09.008

Garceau, C., \& Ronis, S. T. (2017). The interface between young adults' religious values and their sexual experiences before age 16. The Canadian Journal of Human Sexuality, 26(2), 142-150. https://doi.org/10.3138/cjhs.262-a6

García-Gómez, B., García-Cruz, E., Bozzini, G., Justo-Quintas, J., García-Rojo, E., Alonso-Isa, M., \& Romero-Otero, J. (2017). Sexual sat- isfaction: An opportunity to explore overall health in men. Urology, 107, 149-154. https:// doi.org/10.1016/j.urology.2017.06.031

Giner-Sorolla, R., Bosson, J. K., Caswell, T. A., \& Hettinger, V. E. (2012). Emotions in sexual morality: Testing the separate elicitors of anger and disgust. Cognition \& emotion, 26(7), 1208-1222. https://doi.org/10.1080/0269993 1.2011 .645278

Gouveia, V. V., Milfont, T. L., Da Fonseca, P. N., \& de Miranda Coelho, J.A. P. (2009). Life satisfaction in Brazil: Testing the psychometric properties of the satisfaction with life scale (swLS) in five Brazilian samples. Social Indicators Research, 90(2), 267277. https://doi.org/10.1007/s11205-008-9257-0

Greene, D. C., \& Britton, P. J. (2013). The influence of forgiveness on lesbian, gay, bisexual, transgender, and questioning individuals' shame and self-esteem. Journal of Counseling \& Development, 91(2), 195-205. https://doi. org/10.1002/j.1556-6676.2013.00086.x

Heilborn, M. L. (2009). In the fabric of Brazilian sexuality. In I C. E. Bose \& M. Kim (Orgs.). Global Gender Research: Transnational Perspectives (pp. 239-248). Albany, NY: Routledge Publishers.

Heilborn, M. L., \& Cabral, C. D. S. (2013). Youth, gender and sexual practices in Brazil. Psicologia \& Sociedade, 25(SPE), 33-43. https://doi. org/10.1590/S0102-71822013000500005

Hewitt-Stubbs, G., Zimmer-Gembeck, M. J., Mastro, S., \& Boislard, M. A. (2016). A longitudinal study of sexual entitlement and self-efficacy among young women and men: Gender differences and associations with age and sexual experience. Behavioral Sciences, 6(1), 1-14. https://doi.org/10.3390/bs6010004

Horne, S., \& Zimmer-Gembeck, M. J. (2006). The female sexual subjectivity inventory: Development and validation of a multidimensional inventory for late adolescents and emerging adults. Psychology of Women Quarterly, 30(2), 125-138. https://doi. org/10.1111/j.1471-6402.2006.00276.x 
Hutz, C. S., \& Zanon, C. (2011). Revisão da adaptação, validação e normatização da escala de autoestima de Rosenberg: Revision of the adaptation, validation, and normatization of the Roserberg self-esteem scale. Avaliação Psicológica, 10(1), 41-49. Recuperado de http:// pepsic.bvsalud.org/scielo.php?script $=$ sci_arttext\&pid=S1677-04712011000100005\&1$\mathrm{ng}=\mathrm{pt} \& \mathrm{tlng}=\mathrm{pt}$

Impett, E. A. \& Peplau, L. A. (2003). Sexual compliance: Gender, motivational, and relationship perspectives. Journal of Sex Research, 40(1), 87-100. https://doi.org/10.1080/00224490309552169

Koenig, H., Parkerson Jr, G. R., \& Meador, K. G. (1997). Religion index for psychiatric research: a 5-item measure for use in health outcome studies. American Journal of Psychiatry, 154, 885-886.

Marshal, M. P., Dietz, L. J., Friedman, M. S., Stall, R., Smith, H. A., McGinley, J., ... Brent, D. A. (2011). Suicidality and depression disparities between sexual minority and heterosexual youth: A meta-analytic review. Journal of adolescent health, 49(2), 115-123. https://doi.org/10.1016/j. jadohealth.2011.02.005

Martin, K. A. (1996). Puberty, sexuality, and the self: Boys and girls at adolescence. New York, NY: Psychology Press.

Mastro, S. \& Zimmer-Gembeck, M. J. (2015). Let's talk openly about sex: Sexual communication, self-esteem and efficacy as correlates of sexual well-being. European Journal of Developmental Psychology, 12(5), 579-598. https:// doi.org/10.1080/17405629.2015.1054373

Michaud, P. A., Suris, J. C., \& Deppen, A. (2006). Gender-related psychological and behavioural correlates of pubertal timing in a national sample of Swiss adolescents. Molecular and cellular endocrinology, 254, 172-178. https://doi. org/10.1016/j.mce.2006.04.037

Moreau, C., Trussell, J., \& Bajos, N. (2013). Religiosity, religious affiliation, and patterns of sexual activity and contraceptive use in France. The
European journal of contraception \& reproductive health care: The official journal of the european society of contraception, 18(3), 168-180. https://doi.org/10.3109/13625187.2013.777829

Moreira-Almeida, A., Peres, M. F., Aloe, F., Lotufo Neto, F., \& Koenig, H. G. (2008). Portuguese version of Duke Religious Index: DUREL. Revista de Psiquiatria Clínica, 35, 31-32. https:// doi.org/10.1590/S0101-60832008000100006

Pompili, M., Lester, D., Forte, A., Seretti, M. E., Erbuto, D., Lamis, D. A., \& Girardi, P. (2014). Bisexuality and suicide: A systematic review of the current literature. The Journal of Sexual Medicine, 11(8), 1903-1913. https://doi.org/10.1111/jsm.12581

Robinson, K., \& Davies, C. (2008). Docile bodies and heteronormative moral subjects: Constructing the child and sexual knowledge in schooling. Sexuality \& Culture, 12(4), 221-239. https://doi. org/10.1007/s12119-008-9037-7

Sánchez, F. L. (2009). La educación sexual. Madrid: Biblioteca Nueva.

Semenyna, S. W., Belu, C. F., Vasey, P. L., \& Honey, P. L. (2018). Not straight and not straightforward: The relationships between sexual orientation, sociosexuality, and dark triad traits in women. Evolutionary Psychological Science, 4(1), 24-37. https://doi.org/10.1007/ s40806-017-0111-y

Silva, A. D., do Céu Taveira, M., Marques, C., \& Gouveia, V. V. (2015). Satisfaction with life scale among adolescents and young adults in Portugal: Extending evidence of construct validity. Social Indicators Research, 120(1), 309-318. https:// doi.org/10.1007/s11205-014-0587-9

Taunay, T. C. D., Gondim, F. D. A. A., Macedo, D. S., Moreira-Almeida, A., Gurgel, L. D. A., Andrade, L. M. S., \& Carvalho, A. F. (2012). Validação da versão brasileira da escala de religiosidade de Duke (DUREL). Revista de Psiquiatria Clínica, 39(4), 130-135.

Tolman, D. L., \& Tolman, D. L. (2009). Dilemmas of desire: Teenage girls talk about sexuality. Boston, MA: Harvard University Press. 
Woo, J. S., Morshedian, N., Brotto, L. A., \& Gorzalka, B. B. (2012). Sex guilt mediates the relationship between religiosity and sexual desire in East Asian and Euro-Canadian college-aged women. Archives of Sexual Behavior, 41(6), 1485-1495. https://doi.org/10.1007/s10508012-9918-6

World Health Organization. (2004). Sexual health-a new focus for wHO. Progress in reproductive health research, 67(3), 1-8.

Zaleski, E. H., \& Schiaffino, K. M. (2000). Religiosity and sexual risk-taking behavior during the transition to college. Journal of Adolescence, 23(2), 223-227. https://doi.org/10.1006/ jado.2000.0309

Zimmer-Gembeck, M. J. (2013). Young females' sexual self-efficacy: Associations with personal autonomy and the couple relationship.
Sexual health, 10(3), 204-210. https://doi. org/10.1071/SH12139

Zimmer-Gembeck, M. J., \& French, J. (2016). Associations of sexual subjectivity with global and sexual well-being: A new measure for young males and comparison to females. Archives of sexual behavior, 45(2), 315-327. https://doi. org/10.1007/s10508-014-0387-y

Zimmer-Gembeck, M. J., Ducat, W. H., \& Boislard-Pepin, M. A. (2011). A prospective study of young females' sexual subjectivity: Associations with age, sexual behavior, and dating. Archives of sexual behavior, 40(5), 927-938. https://doi. org/10.1007/s10508-011-9751-3

Zivony, A., \& Saguy, T. (2018). Stereotype deduction about bisexual women. The Journal of Sex Research, 55(4-5), 666-678. https://doi.org/ $10.1080 / 00224499.2018 .1437116$ 